\title{
Formation of health culture in the context of digitalization of education
}

\author{
S.I. Kramskoy ${ }^{1 *}$, I.A. Amelchenko², and D.E. Egorov ${ }^{3}$ \\ ${ }^{1}$ V.G. Shukhov Belgorod State Technological University, Belgorod, Russia \\ ${ }^{2}$ V.G. Shukhov Belgorod State Technological University, Belgorod, Russia \\ ${ }^{3}$ Moscow Polytechnic University, Moscow, Russia
}

\begin{abstract}
The article studies the issues related to the use of digital technologies in organization of the educational process of distance learning and testing the quality of the digested educational material in the disciplines of Physical Culture and Sports. The advantages and disadvantages of distance learning organization are revealed. The problems of increasing students' involvement into arranging independent wellness classes are analyzed. The developed electronic information and educational system is an integral part of the program for health culture formation in the context of digitalization of education. The components of the module allow you to distantly teach and test the quality of the digested educational material, as well as to ensure the control of students' fitness shape. The use of modern tools of digital technologies helps to increase students' interest in their classes.
\end{abstract}

\section{A problem statement}

Nowadays digital technologies are widely used in various spheres of life including management of energy, finance, defense and other vital areas of life and national security.

If we try to apply the term "digitalization" to educational activities, then digitalization is an introduction and use of digital technologies in the educational process in order to improve its quality and efficiency, making it accessible at any time and regardless of the place of stay, as well as more capacious and meaningful.

Modern digital educational environment is a promising and dynamically developing project in the field of education $[1,2]$. The main advantages of the electronic educational environment are:

- $\quad$ freedom of access to training resources;

- optimization of costs and time consumption related to students' and teachers' training due to the absence of time expenditures related to getting to the place of study and back to the place of residence, as well as the ability to receive education on the job using the Internet;

- flexibility of training: the student is able to choose the duration and sequence of studying materials by himself, building the learning process according to his capabilities.

${ }^{*}$ Corresponding author: prof-ped.gpa@mail.ru 
The division of the e-course content into modules makes it easier to find necessary materials;

- competence, quality, effectiveness of education: e-courses are created by a team of specialists, the electronic version of educational materials is constantly updated in accordance with trends in normative and methodological fields;

- in order to study it is necessary to have a computer, a tablet or a smartphone with Internet access;

- a variety of forms of learning: interactive lectures, game simulators, interactive tests, virtual laboratory and practical works, modeling of processes that simulate reality, etc;

- students save time and money spent on trip to and from the place of study during classroom learning;

- modern educational products are also changing in accordance with the changes in legislation and trends in society;

- realization of the need for self-study and continuous professional self-improvement. In modern society the ability to work independently plays an important role. When working online an increase in the share of independent learning ensures that students develop the essential skills of searching for the necessary material, studying and interpreting documents, etc [3-9].

The momentum in development of electronic educational programs is determined by complex epidemiological situation. The need to continue the educational process distantly in the conditions of self-isolation prompted the search for new methodological approaches while planning classes [10].

Modern technologies of electronic learning, which are gaining popularity, are becoming more and more required in the educational process in the disciplines of Physical Culture and Sports. In modern times of mass physical culture there is an urgent need to strengthen the recreational component that is determined by a fairly high level of general morbidity of the Russian population including young people. At the universities the task of students' health formation and promotion is assigned to the Departments of Physical Education and Sports. The functioning of these university structural units has certain peculiarities depending on the material and technical conditions, personnel potential, institution's profile and the contingent of those engaged in studying. These features inevitably affect the specifics of the organization of work with students in the disciplines of Physical Culture and Sports. It is relevant to search for and implement effective developments aimed at promoting students' health in relation to a particular educational institution.

\subsection{The objective of the work}

Purpose of the conducted research - development of mechanisms for implementation of the program for health culture formation in the educational process with the use of digital technologies.

\section{Results of the research}

Research methods: analysis of literary sources, sociological survey, pedagogical experiment, pedagogical observations. The research was conducted at the Department of Physical Education and Sports of Belgorod State Technological University named after V.G. Shuhov. According to the questionnaire developed by the authoring team, 300 students of 1-3 courses were interviewed. In the course of student survey it was found out that students are more willing to be engaged in distance learning in comparison with the traditional training system. 
The obtained survey results coincided with the data [11-13] and are determined by the following factors:

- $\quad$ absence of time and money expenditures related to transport;

- $\quad$ ability to keep your own schedule of the day;

- more time allocated to educational activities and personal interests by reallocating

time for transportation to and from the educational institution;

- teachers provide electronic versions of educational materials;

- In distance learning, students are grouped together through various messengers, which facilitates communication and allows for more active exchange of educational materials and test results.

At the Department of Physical Education and Sports of BSTU named after V.G. Shukhov an educational technology was developed that allows us not only to teach and test the quality of the digested educational material online, but also to provide methodological support for the training process in sport.

According to the authors [14], to ensure the educational process, we need constant access to electronic educational resources and systems for monitoring the quality of material comprehension, where students can study the necessary material and assess the quality of material comprehension independently. This can also be applied to Physical Education, which makes it possible to see the performance of motor actions in accordance with the reference technique or to study a set of exercises, as well as to track the dynamics of the level of physical qualities development. A part of the issues (formation of necessary amount of knowledge and control of knowledge level) is solved through the resources of Urite and Google Forms including the testing system proposed by these platforms.

Teachers of the Department of Physical Education and Sports of BSTU named after V.G. Shukhov used Google Forms to test the level of knowledge at the end of the semester. In this case the testing excluded the human factor, the results were obtained immediately after the end of testing. According to the results of the survey, students were more willing to take the test online, but the testing revealed the need to limit the number of attempts to pass the tests, which is due to the students' attempts to find the right answer when they obviously do not know it.

However, there were also students who wanted to take the test face-to-face with the teacher that was explained by the possibility to correct the answer in case of inaccuracy. The automated system will mark this answer as incorrect, while the teacher can correct it or give a suggestive answer. As a result of such communication, the chance to get the desired score increases, while the machine version of the test will not accept the answer.

According to the results of the research carried out by a number of specialists, who conducted their studies in the field of digital education [15-18], as well as their own analysis [19], it can be concluded that the educational environment in the disciplines of Physical Culture and Sports should include a number of components:

- educational module (multimedia lectures, electronic textbooks), during the learning of which the necessary amount of knowledge is formed;

- unit with demonstration of the reference techniques of performing physical actions (video content is prepared by the academic teaching staff of the department or used as it is provided on the Internet);

- unit where well-being, mode and quality of meals, as well as duration of sleep are recorded, i.e. it is necessary to keep a diary of self-control

- the next unit is an application that provides recommendations on the volumes and intensity of motor activity.

At BSTU named after V.G. Shukhov the academic teaching staff of the department developed a media course, which consists of lectures, presentations and a module that tests the level of comprehension of the educational material under study. 
The developed testing module includes test tasks that the student has to complete using a limited number of attempts, as well as questions that the test taker has to answer within a limited amount of time (the number of attempts to answer questions is limited, which does not allow students to select the correct answer if they do not know it).

In the course of conducting online surveys it is necessary to limit the response time since during the survey students turn to Internet sources and do not show their knowledge, which does not contribute to the formation of necessary volume and depth of knowledge of the material under study.

The testing unit also includes control questions, where students are asked to answer control questions covering the studied topics.

The practical part of the classes, where students had to perform sets of exercises, was controlled by diaries of their self-control and video files with the exercises performed that were sent to the e-mail of the student group's tutor. In the course of the study there arose a question about the reliability of the data concerning the completion of the task sent by students. The survey was conducted by questionnaire method and the questionnaire was completely anonymous. As for the data about the respondents, gender, age, course and place of residence without its name, only with indication: city, settlement or village, were required. Sending a diary of self-control was not a problem, while not everyone was able to shoot or send a video file due to either the absence of a person who could shoot exercises being performed or limited Internet traffic.

At BSTU named after V.G. Shukhov in order to increase the informativeness and reliability of the data provided and to create a positive motivational sphere at the classes of elective disciplines in Physical Culture and Sports, an experiment was conducted, in which 70 boys and girls of the 3rd year of the main educational department took part.

In order to improve control over the data provided by students, we created a group that trained distantly with heart rate monitors connected to computers that controlled the physical load during classes and tracked the distance covered during the class.

Those, who did not have a heart rate monitor, used a heart sensor that was "attached" to a smartphone. For their smartphones the students chose applications, in which it was possible to track the distance covered by the student during classes. Most students selected such programs as Nike Training Club, Map My Fitness, Charity Miles.

When organizing and holding classes, a standard system was built: recommendations on physical load and its dynamics during the class, load control and recovery rate control. At the end of the lessons the teacher gave recommendations concerning body recovery after exercises and dynamics of the load during the classes, which were based on the information received.

At the beginning of classes in distant mode students were given recommendations on the dosage of physical loads. At the end of classes each student could see the dynamics of heart rate in the process of performing the load during the lesson, throughout the day, week, month. The possibilities were limited only by the capabilities of the software and the functionality of the electronic devices [20].

The choice of cyclic sports is determined by the lack of sportsgrounds for sports game, as well as quarantine measures.

During the classes the following tasks were set: increasing motor activity, relieving psychological stress caused by prolonged stay in confined space, developing endurance, dexterity and coordination abilities.

Thus, in the groups training with electronic heart rate monitors and heart sensors students showed great interest in the classes and, as an evidence, greater number of students sent a diary of their self-control and a track of the distance covered. While in the groups that trained without any gadgets the number of students who sent a diary of their selfcontrol was lower. When identifying the reasons for this situation it turned out that students 
were more interested in working with heart rate monitors and cardio sensors because it is possible to monitor the pulse, track its dynamics and heart rate. According to the students, on the Internet it was interesting for the girls to train in compliance with the loads causing a decrease in body weight. This motive was found to be predominant among the others by $70 \%$ of the girls. For the others the main motive was to get a credit. Besides, family members joined those students who trained with electronic gadgets, which also increased the interest in the classes, according to the students. The diaries of self-control contained a body weight line that students had to fill in. However, in this case there was a problem: only $13 \%$ of students had scales. As a result of conversations with students about the need for a healthy lifestyle and about weight control as a part of it, a significant number of students purchased scales independently or in groups.

Another factor that increased the responsibility for provided data was the condition to send the track of the covered distance along with the diary of self-control. This condition motivated students' responsibility when sending data. Moreover, the authors did not restrict students in the form of sports activities. It was one of the cyclic types (running, cycling, or swimming). When choosing swimming it was necessary to provide a swimming pool membership. In this case the swimming distance depended on the ability to swim.

Electronic gadgets used to control the load contributed to the formation of the necessary amount of knowledge.

In order to form the necessary amount of knowledge the academic teaching staff created theoretical multimedia course in Physical Culture. We also recommended the Urite library, E library and a number of other resources listed above. The main problem for university teachers and students is the commercial basis of the provision of educational materials. Not all students can pay for the use of electronic library content.

When choosing or recommending Internet educational material to students it is necessary to focus on its content and quality. The educational material posted on the Internet contains not only textbooks, articles, but also educational films and video clips. The latter are the most popular, since there are quite a lot of them on Urite and YouTube, but you need to pay close attention to their content when making recommendations for studying them.

\section{Conclusions}

The developed electronic information and educational system is an integral part of the program for the formation of health culture in the context of digitalization of education. The components of the module allow you to distantly train and test the quality of the digested educational material, as well as to ensure the control of students' fitness shape. The use of modern tools of digital technologies helps to increase students' interest in classes.

\section{References}

1. T.E. Lebedeva, The use of a complex of information technologies in a practical lesson at a university as a means of improving the quality of teacher training, 22 (2008)

2. E.V. Yakovleva, Distance learning in an information educational environment, Young Scientist, 10, 69-71 (2018)

3. E.V. Belyaeva, E.F. Moskaleva, Designing new teaching technologies based on software pedagogical tools, Higher education today, 11, 43-46 (2011)

4. E.V. Yashchuk, E.Yu. Zankova, Continuous pedagogical education in the context of the introduction of e-learning, 9, 167 (2015) 
5. T.E. Lebedeva, Information technologies in practical classes at the university, Higher education today, 8, 49-51 (2007)

6. T.E. Lebedeva, N.E. Okhotnikova, E.A. Potapova, Electronic educational environment of the university: requirements, opportunities, experience and prospects of use, Internet journal "World of Science", 4 (16), 56-58 (2016)

7. I.V. Robert, Theory and methodology of informatization of education (psychological, pedagogical and technological aspects), 356 (2010).

8. E.K. Samerkhanova, Network interaction between teacher and students as the main condition for creating a single information and educational space, School of the Future, 6, 65-68 (2011)

9. E.V. Gavrishova, A.S. Grachev, A.A. Tretyakov, The use of information and communication technologies in the organization of additional health-improving activities of students, taking into account the type of motivation, Theory and practice of physical culture, 1(979), 44-48 (2020)

10. M.G. Balykhin, E-learning and its role in education without borders, Bulletin of the Peoples' Friendship University of Russia. Series: educational issues: languages and specialties, 4, 68 (2008)

11. S.E. Cherkezov, Basic Models for the Development of Electronic Education as an Innovative System, Izvestia of the Southern Federal University. Pedagogical sciences, 3, 206 (2009)

12. A.P. Shmakova, E.V. Belyaeva, Distance learning as a way of organizing independent work of bachelors, Theory and practice of social development, 10, 190 (2020)

13. A.N. Safronova, Theory and methodology for the formation of socially significant skills of self-preservation of health among students in a real-virtual educational environment of the university, Modern problems of science and education, 5 (2018)

14. A.N. Veryaskina, Modern technological revolution: methodological and didactic aspects, Modern high technologies, 9, 110 (2015)

15. A.D. Afrikyan, A.A. Ermakova, I.A. Tarasova, Electronic textbook for correspondence courses in technical universities as an element of distance education, Izvestia of the Volgograd State Technical University. Series: new educational systems and technologies of teaching at the university, 5(5), 13-14 (2008)

16. I.A. Stetsenko, E.V. Yashchuk, Technologies of e-learning in the system of continuous pedagogical education (from the experience of the laboratory of problems of informatization of education), Bulletin of the Siberian Institute of Business and Information Technologies, 1(9), 95 (2014)

17. I.P. Ushakov, O.I Milkova, Experience in the development of electronic teaching aids for distance learning for the main educational programs of higher professional education, Innovative development of the economy. Scientific Consulting Center, 3(9), 100-101 (2012)

18. N.V. Lomovtseva, Formation of the readiness of university teachers to use distance learning technologies, 12 (2009)

19. S.I. Kramskoy, I.A. Amelchenko, D.E. Egorov, Interdisciplinary approach to health life style model development in students, Advances in Social Science, Education and Humanities Research (ASSEHR), 312, 23 (2018)

20. S.I Kramskoy, D.E. Egorov, I.A. Amelchenko, Functional state assessment during physical education classes by experimental method, Advances in social science, education and humanities research (ASSEHR), 97, 355 (2017) 